\title{
Beneficios de la lactancia materna
}

\section{Carta al Editor}

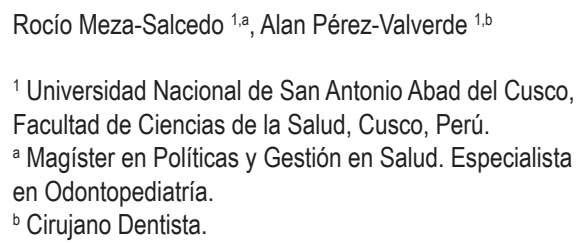

\section{Correspondencia:}

Rocío Meza Salcedo: mezasalcedorocio@gmail.com Av. Camino Real 110. Urb. Vallecito. Cusco Perú. ORCID: 0000-0003-0538-9177

\section{Benefits of breastfeeding}

\section{Sr. Editor.}

Debido a la pandemia por COVID-19 se puso en duda la importancia de la lactancia materna, por la probabilidad de contagio al bebé o recién nacido; por ello plantearemos la importancia de los beneficios, priorizando al ser humano futuro y tratando de encontrar un equilibrio entre salud bucal, salud general y riesgos generales, siempre con un enfoque multidisciplinario y basada en evidencias como:

- Disminución en el bebé de sobrepeso y obesidad en un 13\%, de la diabetes en 35\%; debido a que a partir de los 6 meses la composición de la leche cambia epigenéticamente, pues hay una reducción de tantas calorías y grasas para no sobrecargar el cuerpo del bebé e influenciado por la dieta de la madre ${ }^{1}$.

- Mayor coeficiente intelectual en 2,6, independiente del coeficiente intelectual materno, debido a que el contacto piel a piel que ofrece la lactancia materna estimula el desarrollo y producción de oxitocinas (hormona del amor y del desarrollo) y porque la leche materna es fuente natural del DHA (ácido docosahexaenoico) que ayuda a mayores conexiones sinápticas; ya que el $80 \%$ del desarrollo cognitivo se forma hasta los 2 años de edad ${ }^{1}$.

- Reducción de riesgo de otitis media aguda en un 43\% debido a que se estimulan muchos músculos como el tensor del paladar membranoso que permite el pasaje con la tuba auditiva, estimulado durante la lactancia, en el proceso de maduración ${ }^{2}$.

- Disminución de riesgo de leucemia infantil en un 19\% para la lactancia materna exclusiva por lo menos 6 meses, ya que la leche materna contiene inmunoglobulinas, anticuerpos, células madre, linfocitos $\mathrm{T}$ y $\mathrm{B}$
Alan Pérez Valverde: alanpv1983@gmail.com ORCID: 0000-0002-9476-3548

Conflicto de intereses: ninguno.

Fuente de financiamiento: autofinanciado.

Recibido: $15 / 03 / 21$

Aprobado: $12 / 04 / 21$

Publicado: 01/07/21

\section{Coautor:}

(C) Los autores. Este artículo es publicado por la revista Odontología Sanmarquina de la Facultad de Odontología, Universidad Nacional Mayor de San Marcos. Este es un artículo de acceso abierto, distribuido bajo los términos de la licencia Creative Commons Atribución 4.0 Internacional (CC BY 4.0) [https://creativecommons.org/ licenses/by/4.0/deed.es] que permite el uso, distribución y reproducción en cualquier medio, siempre que la obra original sea debidamente citada de su fuente original. 
con bastante potencial epigenético, aunque sin evidencias frente al mecanismo de acción ${ }^{3}$.

- Reduce en 36\% el síndrome de muerte súbita durante el primer año de vida, sabiendo que entre los cuatro y seis meses de vida hay mayor riesgo de asfixias; y propiciando la lactancia materna mejora la respiración nasal, el control de las vías aéreas superiores, reduciendo el riesgo de hipoxias ${ }^{4}$.

- Disminución de infecciones por diarrea en $72 \%$ y neumonía en 57\%, evitando las dos principales causas de muertes en bebés menores de 1 año de edad en América Latina y Caribe; y correlativamente la reducción en defectos del esmalte ya que uno de las causas de estos, son fiebres altas e infecciones ${ }^{5}$.

- En la madre se observa disminución de cáncer de mama en $4,3 \%$ por ańo, cáncer de ovario en $18 \%$ y diabetes tipo 2 en $32 \%$ por mecanismos hormonales, ya que la lactancia mayor de 12 meses actúa como un factor reductor del cáncer; teniendo en consideración que el cáncer de mama continúa siendo muy prevalente y a pesar de ser tratable hay mucha mortalidad por esta causa $^{6}$.

- Reducción de peso de la madre (menores de 35 años), ya que el metabolismo es distinto y asociado a una nutrición adecuada y equilibrada observándose pérdida de peso post parto acelerada, por desgaste calórico, por la oxitocina que provoca la involución uterina mientras se lacte haciendo que se contraiga ${ }^{7}$.

- Además de beneficios como la reducción de maloclusiones entre $51 \%$ y $68 \%$, sobretodo en dentición temporal como predictora de la dentición permanente ${ }^{8}$ en niños amamantados con una adecuada técnica.

Conociendo los beneficios de la lactancia materna exclusiva prolongada y separándolo de cualquier componente genético tanto para el bebé como para la madre, se concluye que es importante divulgar y motivar lo relevante de este acto y así evitar futuras complicaciones para la salud.

\section{Referencias bibliográficas}

1. Horta BL, Loret de Mola C, Victora CG. Breastfeeding and intelligence: a systematic review and meta-analysis. Acta Paediatr. 2015;104(467):14-9. DOI:10.1111/ apa.13139.

2. Bowatte G, Tham R, Allen KJ, Tan DJ, Lau M, Dai X, et at. Breastfeeding and childhood acute otitis media: a systematic review and meta-analysis. Acta Paediatr. 2015;104(467):85-95. DOI: 10.1111/apa.13151

3. Amitay EL, Keinan-Boker L. Breastfeeding and Childhood Leukemia Incidence: A Meta-analysis and Systematic Review. JAMA Pediatr. 2015;169(6):e151025. DOI: $10.1001 /$ jamapediatrics.2015.1025.

4. Hauck FR, Thompson JM, Tanabe KO, Moon RY, Vennemann MM. Breastfeeding and reduced risk of sudden infant death syndrome: a meta-analysis. Pediatrics. 2011;128(1):103-10. DOI: 10.1542/peds.2010-3000.

5. Horta BL, Victora CG, World Health Organization. Short-term effects of breastfeeding: a systematic review on the benefits of breastfeeding on diarrhoea and pneumonia mortality. World Health Organization. 2013. https://apps.who.int/iris/handle/10665/95585

6. Chowdhury R, Sinha B, Sankar MJ, Taneja S, Bhandari N, Rollins N, et al. Breastfeeding and maternal health outcomes: a systematic review and meta-analysis. Acta Paediatr. 2015;104(467):96-113. DOI: 10.1111/ apa.13102.

7. Kramer MS, Kakuma R. Optimal duration of exclusive breastfeeding. Cochrane Database Syst Rev. 2012(8):CD003517. DOI: 10.1002/14651858.

8. Chen XX, Xia B, Ge LH, Yuan JW. Effects of breast-feeding duration, bottle-feeding duration and oral habits on the occlusal characteristics of primary dentition. 2016;48(6):1060-1066. 